\title{
Supporting Information for \\ Efficient pretreatment of wheat straw using novel renewable cholinium ionic liquids to improve enzymatic saccharification
}

\author{
Huan Ren, Min-Hua Zong, Hong Wu, Ning Li,*
}

State Key Laboratory of Pulp and Paper Engineering, School of Food Science and

Technology, South China University of Technology, Guangzhou 510640, China

* Corresponding author

Dr. N. Li, Tel/Fax: +86 202223 6669; Email: lining@ scut.edu.cn

Table S1. Effect of [Ch][Tau] on determination of reducing sugar concentrations by the DNS

\begin{tabular}{ccc} 
& \multicolumn{2}{c}{ method } \\
\hline \multirow{2}{*}{$\begin{array}{c}\text { IL concentration } \\
(\mathrm{mg} / \mathrm{mL})\end{array}$} & \multicolumn{2}{c}{ Glucose concentration $(\mathrm{mg} / \mathrm{mL})$} \\
\cline { 2 - 3 }$-^{\mathrm{a}}$ & Theoretical value & Real value \\
0.40 & 2.00 & 2.10 \\
0.35 & 2.00 & 2.06 \\
0.20 & 2.00 & 2.06 \\
$-\mathrm{a}$ & 2.00 & 2.03 \\
0.70 & 4.00 & 4.03 \\
0.40 & 4.00 & 4.05 \\
0.10 & 4.00 & 4.05 \\
& 4.00 & 4.06 \\
\hline
\end{tabular}

a: without adding IL.

Table S2. $T_{\mathrm{d}}$ of representative ILs

\begin{tabular}{cc}
\hline ILs & $T_{\mathrm{d}}\left({ }^{\circ} \mathrm{C}\right)$ \\
\hline$[\mathrm{Ch}][\mathrm{Tau}]$ & 180,286 \\
{$[\mathrm{Ch}][\mathrm{Lev}]$} & 180 \\
{$[\mathrm{Ch}][\mathrm{Fur}]$} & 215 \\
{$[\mathrm{Ch}]\left[\mathrm{HSO}_{4}\right]$} & 293 \\
\hline
\end{tabular}




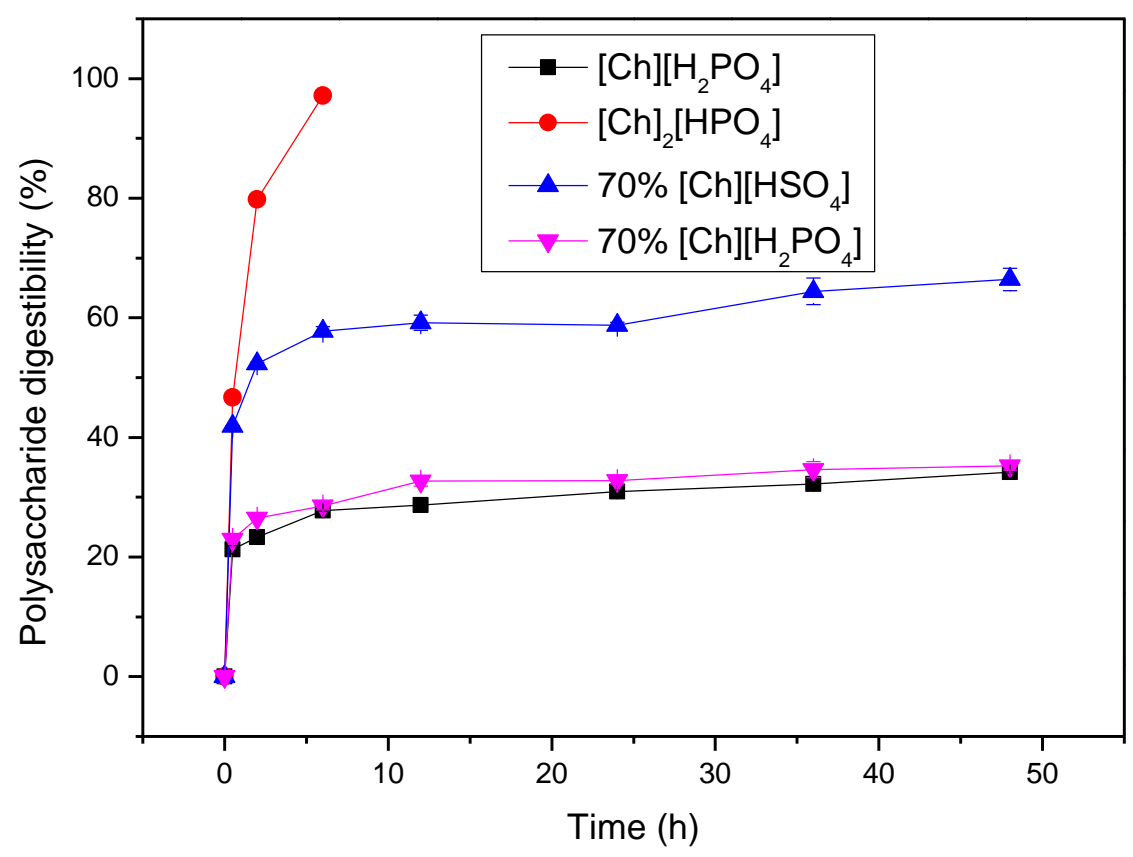

Fig. S1 Time courses of enzymatic hydrolysis of polysaccharides in wheat straw pretreated by ILs. Reaction conditions: $20 \mathrm{mg}$ recovered wheat straw, $7 \mathrm{~mL}$ citrate buffer $\left(50 \mathrm{mmol} \mathrm{L}^{-1}, \mathrm{pH} 4.8\right), 27$ FPU g- ${ }^{-1}$ cellulase, $127 \mathrm{CBU} \mathrm{g}^{-1} \beta$-glucosidase, $0.02 \mathrm{mg} \mathrm{mL}^{-1} \mathrm{NaN}_{3}, 50{ }^{\circ} \mathrm{C}, 200 \mathrm{rpm}$

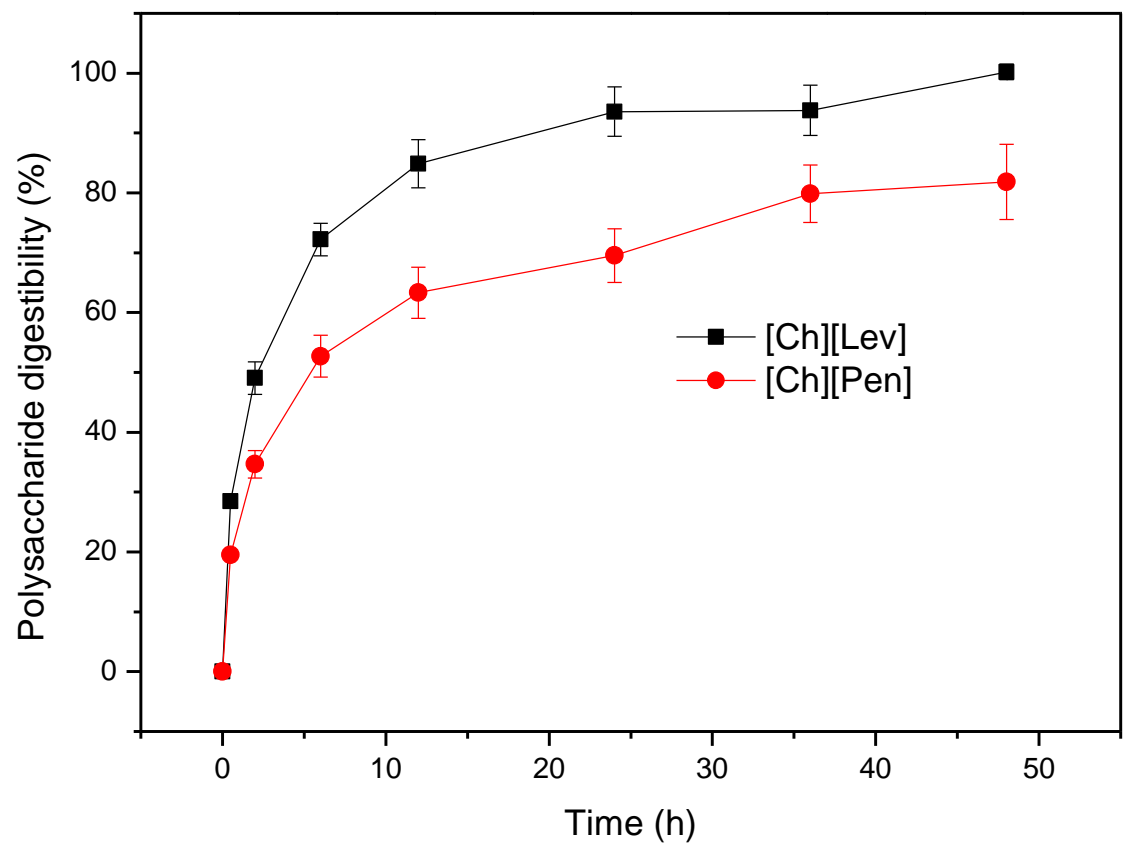

Fig. S2 Time courses of enzymatic hydrolysis of polysaccharides in wheat straw pretreated by ILs. Reaction conditions: $20 \mathrm{mg}$ recovered wheat straw, $7 \mathrm{~mL}$ citrate buffer $\left(50 \mathrm{mmol} \mathrm{L}^{-1}, \mathrm{pH} 4.8\right), 11$ FPU g-1 cellulase, $50 \mathrm{CBU} \mathrm{g}^{-1} \beta$-glucosidase, $0.02 \mathrm{mg} \mathrm{mL}^{-1} \mathrm{NaN}_{3}, 50{ }^{\circ} \mathrm{C}, 200 \mathrm{rpm}$ 

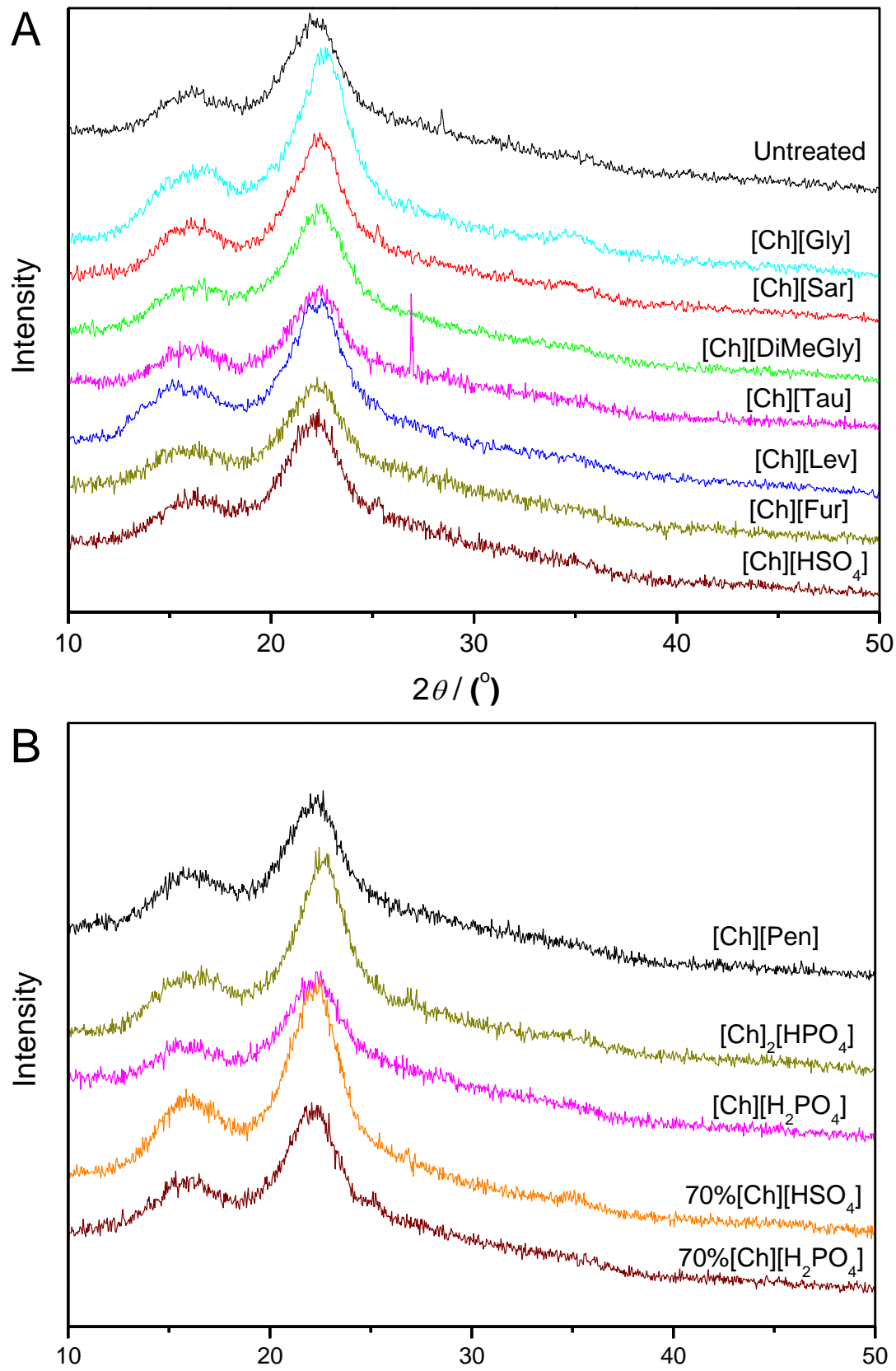

Fig. S3 X-ray diffraction spectra of untreated and IL-pretreated wheat straw 
Table S3. Effect of pretreatment temperature on [Ch][Tau] pretreatment and subsequent enzymatic hydrolysis

\begin{tabular}{|c|c|c|c|c|c|c|c|}
\hline \multicolumn{3}{|c|}{ Pretreatment ${ }^{a}$} & \multicolumn{3}{|c|}{ Composition of residues $(\%)^{b}$} & \multicolumn{2}{|c|}{ Enzymatic hydrolysis of residues ${ }^{c}$} \\
\hline $\begin{array}{c}\text { Temperature } \\
\left({ }^{\circ} \mathrm{C}\right)\end{array}$ & $\begin{array}{l}\text { Lignin } \\
\text { extracted } \\
(\%)\end{array}$ & $\begin{array}{l}\text { Residues } \\
\text { recovery } \\
(\%)\end{array}$ & $\begin{array}{l}\text { Polysacch } \\
\text { arides }\end{array}$ & AIL & ASL & $\begin{array}{c}\text { Initial } \\
\text { saccharification } \\
\text { rate }\left(\mathrm{mg} \mathrm{mL}^{-1} \mathrm{~h}^{-1}\right)\end{array}$ & $\begin{array}{l}\text { Reducing } \\
\text { sugar yield } \\
(\%)\end{array}$ \\
\hline Untreated & 0 & 100 & $60.4 \pm 2.3$ & $15.5 \pm 0.5$ & $1.86 \pm 0.07$ & $0.57 \pm 0.01$ & $24.0 \pm 0.4$ \\
\hline 50 & $14.0 \pm 0.2$ & $77.2 \pm 1.8$ & $66.6 \pm 2.8$ & $17.3 \pm 0.5$ & $1.68 \pm 0.04$ & $0.52 \pm 0.03$ & $21.6 \pm 1.1$ \\
\hline 60 & $16.4 \pm 5.7$ & $73.8 \pm 1.7$ & $68.9 \pm 1.8$ & $17.8 \pm 1.2$ & $1.62 \pm 0.02$ & $0.64 \pm 0.04$ & $33.0 \pm 9.0$ \\
\hline 70 & $33.5 \pm 3.4$ & $66.6 \pm 0.3$ & $69.8 \pm 1.0$ & $15.7 \pm 0.9$ & $1.64 \pm 0.02$ & $0.82 \pm 0.04$ & $63.2 \pm 2.1$ \\
\hline 80 & $53.3 \pm 3.1$ & $60.7 \pm 1.0$ & $72.1 \pm 0.7$ & $11.8 \pm 0.7$ & $1.49 \pm 0.04$ & $0.84 \pm 0.05$ & $74.2 \pm 1.3$ \\
\hline 90 & $65.2 \pm 1.8$ & $55.4 \pm 0.0$ & $73.6 \pm 2.8$ & $9.5 \pm 0.7$ & $1.39 \pm 0.14$ & $0.8 \pm 0.05$ & $73.8 \pm 3.4$ \\
\hline
\end{tabular}

a Samples of wheat straw $(150 \mathrm{mg},<150 \mu \mathrm{m})$ were incubated in [Ch][Tau] IL (3 g) under $\mathrm{N}_{2}$ with stirring at different temperature for $6 \mathrm{~h}$.

${ }^{b}$ Determined via the NREL protocol (LAP version 2008). Polysaccharides content was determined by DNS method.

Results are expressed as a percentage of the residues. AIL, acid-insoluble lignin; ASL, acid-soluble lignin.

${ }^{c}$ Reaction conditions: $20 \mathrm{mg}$ recovered wheat straw, $7 \mathrm{~mL}$ citrate buffer (50 mmol L-1 $\left.\mathrm{pH} 4.8\right), 11 \mathrm{FPU} \mathrm{g}^{-1}$ cellulase, $50 \mathrm{CBU} \mathrm{g}^{-1} \beta$-glucosidase, $0.02 \mathrm{mg} \mathrm{mL}^{-1} \mathrm{NaN}_{3}, 50{ }^{\circ} \mathrm{C}, 200 \mathrm{rpm}$.

Table S4. Effect of pretreatment time on [Ch][Tau] pretreatment and subsequent enzymatic hydrolysis

\begin{tabular}{|c|c|c|c|c|c|c|c|}
\hline \multicolumn{3}{|c|}{ Pretreatment ${ }^{a}$} & \multicolumn{3}{|c|}{ Composition of residues $(\%)^{b}$} & \multicolumn{2}{|c|}{$\begin{array}{c}\text { Enzymatic hydrolysis of residues } \\
c\end{array}$} \\
\hline Time (h) & $\begin{array}{l}\text { Lignin } \\
\text { extracted } \\
(\%)\end{array}$ & $\begin{array}{l}\text { Residues } \\
\text { recovery } \\
(\%)\end{array}$ & $\begin{array}{l}\text { Polysacch } \\
\text { arides }\end{array}$ & AIL & ASL & $\begin{array}{c}\text { Initial } \\
\text { saccharification } \\
\text { rate }\left(\mathrm{mg} \mathrm{mL}^{-1} \mathrm{~h}^{-1}\right)\end{array}$ & $\begin{array}{l}\text { Reducing } \\
\text { sugar yield } \\
(\%)\end{array}$ \\
\hline 1 & $22.4 \pm 0.6$ & $68.2 \pm 5.3$ & $66.8 \pm 0.6$ & $18.3 \pm 1.4$ & $1.44 \pm 0.02$ & $0.62 \pm 0.01$ & $32.4 \pm 2.6$ \\
\hline 2 & $24.3 \pm 2.7$ & $66.5 \pm 2.4$ & $68.2 \pm 0.0$ & $18.3 \pm 1.4$ & $1.42 \pm 0.01$ & $0.65 \pm 0.03$ & $45.0 \pm 1.1$ \\
\hline 4 & $40.4 \pm 6.5$ & $67.6 \pm 2.2$ & $67.6 \pm 1.0$ & $13.8 \pm 1.2$ & $1.42 \pm 0.00$ & $0.69 \pm 0.01$ & $60.7 \pm 4.6$ \\
\hline 6 & $53.5 \pm 4.7$ & $62.6 \pm 2.6$ & $71.2 \pm 1.4$ & $11.5 \pm 0.7$ & $1.36 \pm 0.05$ & $0.85 \pm 0.03$ & $73.0 \pm 5.2$ \\
\hline 8 & $55.1 \pm 0.4$ & $59.6 \pm 0.6$ & $72.6 \pm 4.9$ & $11.8 \pm 0.2$ & $1.23 \pm 0.01$ & $0.69 \pm 0.02$ & $68.0 \pm 0.4$ \\
\hline
\end{tabular}

${ }^{\text {a }}$ Samples of wheat straw $(150 \mathrm{mg},<150 \mu \mathrm{m})$ were incubated in [Ch][Tau] IL (3 g) under $\mathrm{N}_{2}$ with stirring at $80{ }^{\circ} \mathrm{C}$ for different time.

${ }^{b}$ Determined via the NREL protocol (LAP version 2008). Polysaccharides content was determined by DNS method. Results are expressed as a percentage of the residues. AIL, acid-insoluble lignin; ASL, acid-soluble lignin.

${ }^{c}$ Reaction conditions: $20 \mathrm{mg}$ recovered wheat straw, $7 \mathrm{~mL}$ citrate buffer $\left(50 \mathrm{mmol} \mathrm{L}^{-1}, \mathrm{pH} 4.8\right), 11 \mathrm{FPU} \mathrm{g}^{-1}$ cellulase, $50 \mathrm{CBU} \mathrm{g}^{-1} \beta$-glucosidase, $0.02 \mathrm{mg} \mathrm{mL}^{-1} \mathrm{NaN}_{3}, 50{ }^{\circ} \mathrm{C}, 200 \mathrm{rpm}$. 


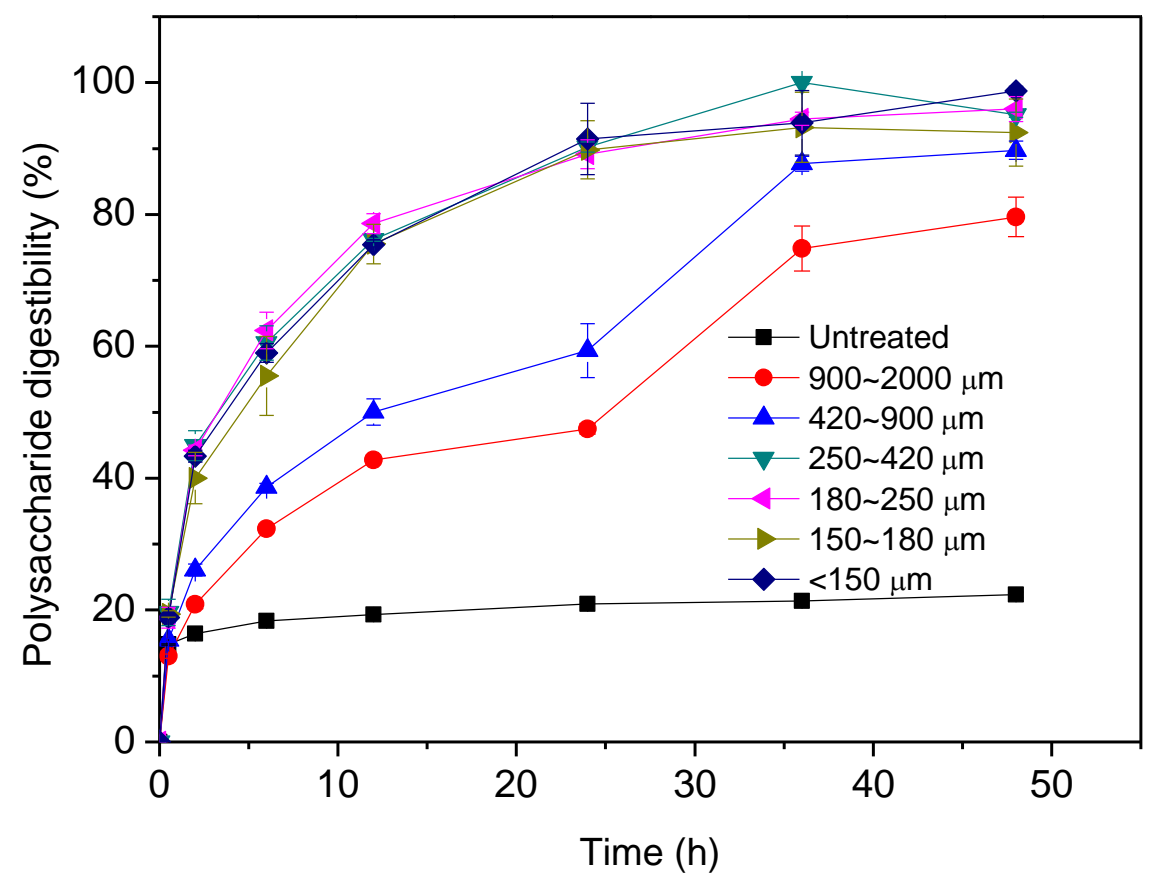

Fig. S4 Time courses of enzymatic hydrolysis of polysaccharides in the IL-pretreated wheat straw with different particle sizes. Reaction conditions: $20 \mathrm{mg}$ recovered wheat straw, $7 \mathrm{~mL}$ citrate buffer $\left(50 \mathrm{mmol} \mathrm{L}^{-1}, \mathrm{pH} 4.8\right), 11 \mathrm{FPU} \mathrm{g}^{-1}$ cellulase, $50 \mathrm{CBU} \mathrm{g}^{-1} \beta$-glucosidase, $0.02 \mathrm{mg} \mathrm{mL}^{-1}$ $\mathrm{NaN}_{3}, 50^{\circ} \mathrm{C}, 200 \mathrm{rpm}$

Table S5. Effect of water content on furfural production during aqueous [Ch][Tau] pretreatment

\begin{tabular}{|ccc|}
\hline & \multicolumn{2}{c|}{ Furfural yield (\%) } \\
\cline { 2 - 3 } Water content (wt \%) & Xylose & Xylan \\
\hline 0 & 0 & 0 \\
20 & 0 & 0 \\
50 & $0.4 \pm 0.1$ & 0 \\
80 & 0 & 0 \\
\hline
\end{tabular}

Pretreatment and analytic conditions: $39.9 \mathrm{mg}$ xylose or $35.1 \mathrm{mg}$ xylan was incubated in $3 \mathrm{~g}$ of aqueous [Ch][Tau] solutions under $\mathrm{N}_{2}$ at $80{ }^{\circ} \mathrm{C}$ for $6 \mathrm{~h}$. Then, furfural was analyzed by reversed phase HPLC on an Eclipse Plus C18 column $(4.6 \mathrm{~mm} \times 250 \mathrm{~mm}, 5 \mu \mathrm{m}$, Agilent, USA) using a Waters 1525 pump and a 2489 UV Detector. The mobile phase was a mixture of methanol and water $(20 / 80, \mathrm{v} / \mathrm{v})$ with a flow rate of $1 \mathrm{~mL} / \mathrm{min}$. The detection wavelength was $285 \mathrm{~nm}$. The retention time of furfural was $6.5 \mathrm{~min}$.

NMR data and spectra of ILs

${ }^{1} \mathrm{H}$ NMR spectra of ILs were recorded in $\mathrm{D}_{2} \mathrm{O}$ at $600 \mathrm{MHz}$ by a NMR spectrometer at $600 \mathrm{MHz}$ (Bruker AVANCE III HD 600, Germany). 


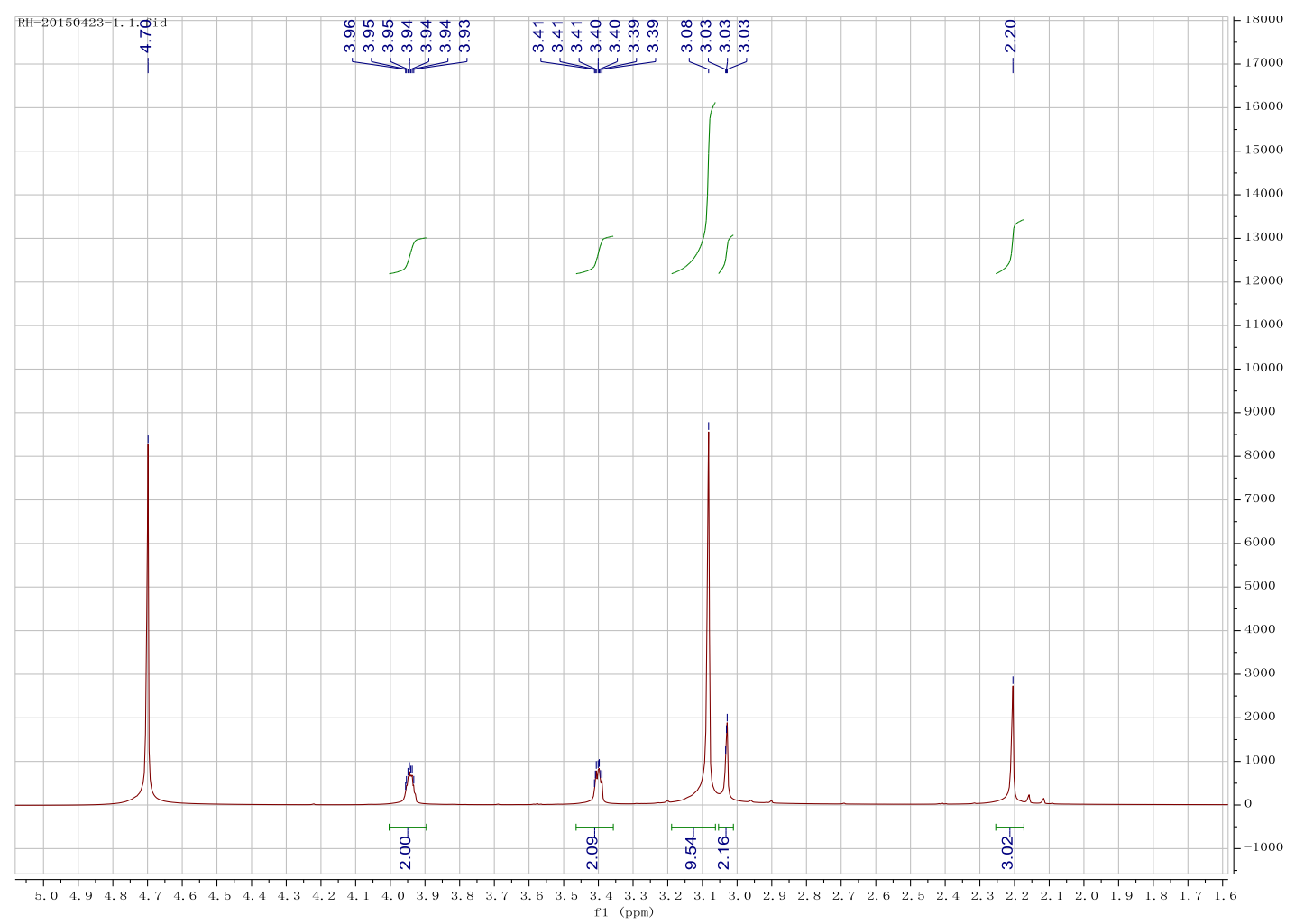

[Ch][Sar]. ${ }^{1} \mathrm{H}$ NMR $\left(600 \mathrm{MHz}, \mathrm{D}_{2} \mathrm{O}\right) \delta: 2.20\left(\mathrm{~s}, 3 \mathrm{H}, \mathrm{CH}_{3}\right), 3.03\left(\mathrm{br} \mathrm{s}, 2 \mathrm{H}, \mathrm{CH}_{2}\right), 3.08\left(\mathrm{~s}, 9 \mathrm{H}, \mathrm{CH}_{3}\right.$, $\left.\mathrm{CH}_{3}, \mathrm{CH}_{3}\right), 3.39-3.41\left(\mathrm{~m}, 2 \mathrm{H}, \mathrm{CH}_{2}\right), 3.93-3.96\left(\mathrm{~m}, 2 \mathrm{H}, \mathrm{CH}_{2}\right)$

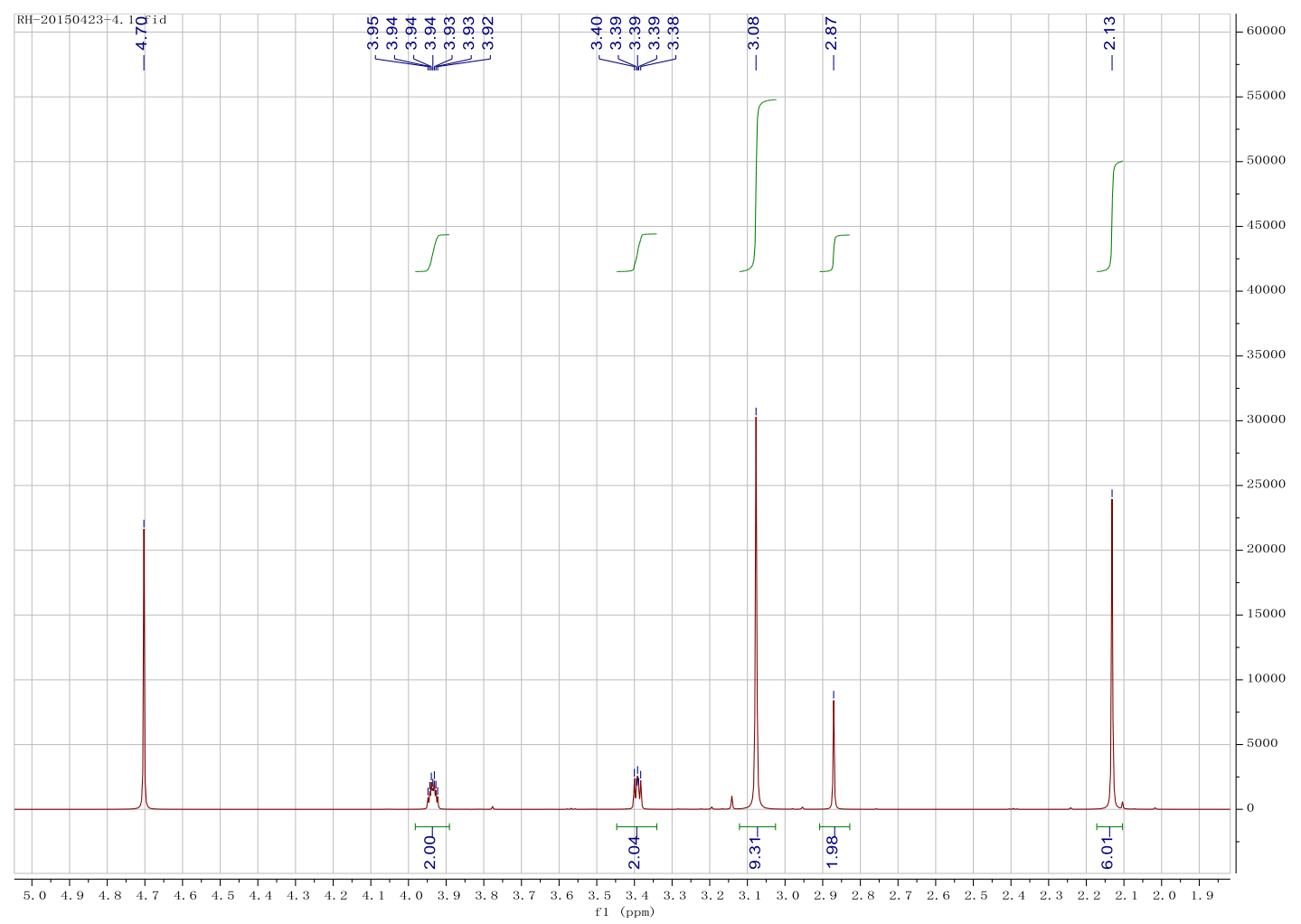

[Ch][DiMeGly]. ${ }^{1} \mathrm{H}$ NMR (600 MHz, $\mathrm{D}_{2} \mathrm{O}$ ): 2.13 (s, 6H, $\left.\mathrm{CH}_{3}, \mathrm{CH}_{3}\right), 2.87$ (s, 2H, $\mathrm{CH}_{2}$ ), 3.08 (s, 9H, $\left.\mathrm{CH}_{3}, \mathrm{CH}_{3}, \mathrm{CH}_{3}\right), 3.38-3.40\left(\mathrm{~m}, 2 \mathrm{H}, \mathrm{CH}_{2}\right), 3.92-3.95\left(\mathrm{~m}, 2 \mathrm{H}, \mathrm{CH}_{2}\right)$ 


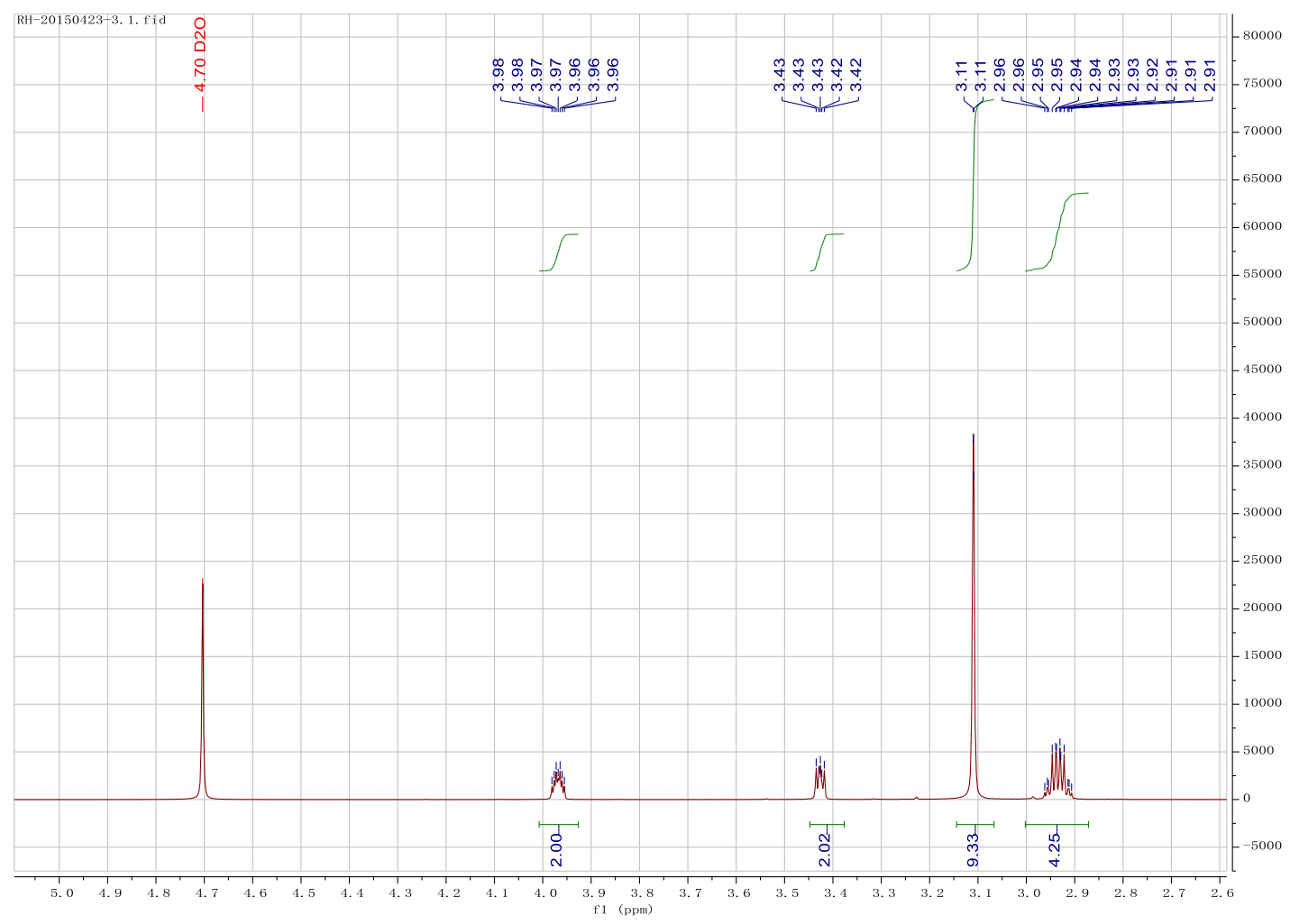

[Ch][Tau]. ${ }^{1} \mathrm{H}$ NMR (600 MHz, $\mathrm{D}_{2} \mathrm{O}$ ): $2.91-2.96$ (m, 4H, $\left.\mathrm{CH}_{2}, \mathrm{CH}_{2}\right), 3.11$ (s, 9H, $\mathrm{CH}_{3}, \mathrm{CH}_{3}, \mathrm{CH}_{3}$ ), $3.42-3.43\left(\mathrm{~m}, 2 \mathrm{H}, \mathrm{CH}_{2}\right), 3.96-3.98\left(\mathrm{~m}, 2 \mathrm{H}, \mathrm{CH}_{2}\right)$

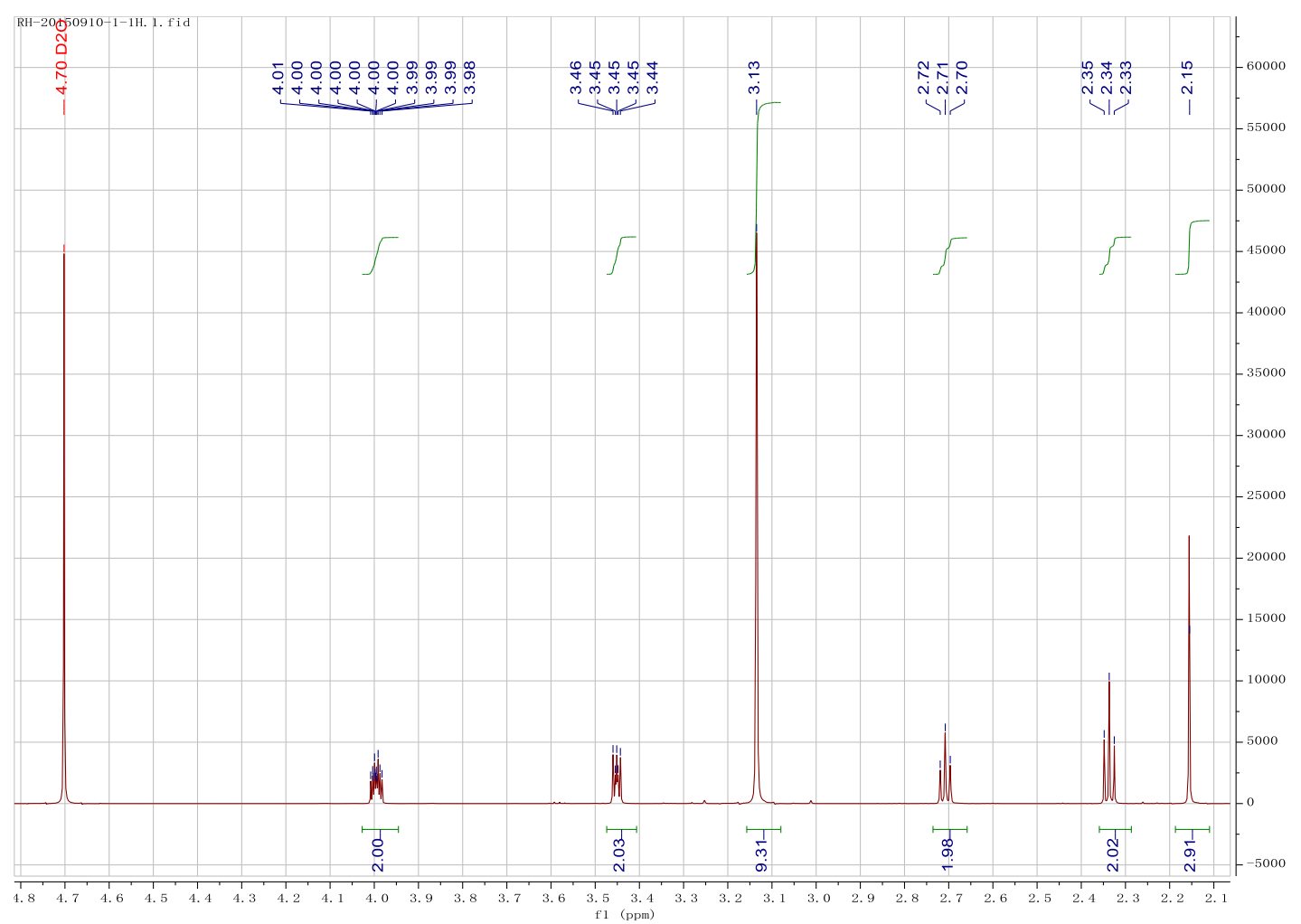

[Ch][Lev]. ${ }^{1} \mathrm{H}$ NMR $\left(600 \mathrm{MHz}, \mathrm{D}_{2} \mathrm{O}\right): 2.15\left(\mathrm{~s}, 3 \mathrm{H}, \mathrm{CH}_{3}\right), 2.34\left(\mathrm{t}, J=6.0 \mathrm{~Hz}, 2 \mathrm{H}, \mathrm{CH}_{2}\right), 2.71(\mathrm{t}, J=$ $6.0 \mathrm{~Hz}, 2 \mathrm{H}, \mathrm{CH}_{2}$ ), 3.13 (s, 9H, $\mathrm{CH}_{3}, \mathrm{CH}_{3}, \mathrm{CH}_{3}$ ), 3.44- 3.46 (m, 2H, $\mathrm{CH}_{2}$ ), 3.98-4.01 (m, 2H, $\mathrm{CH}_{2}$ ) 


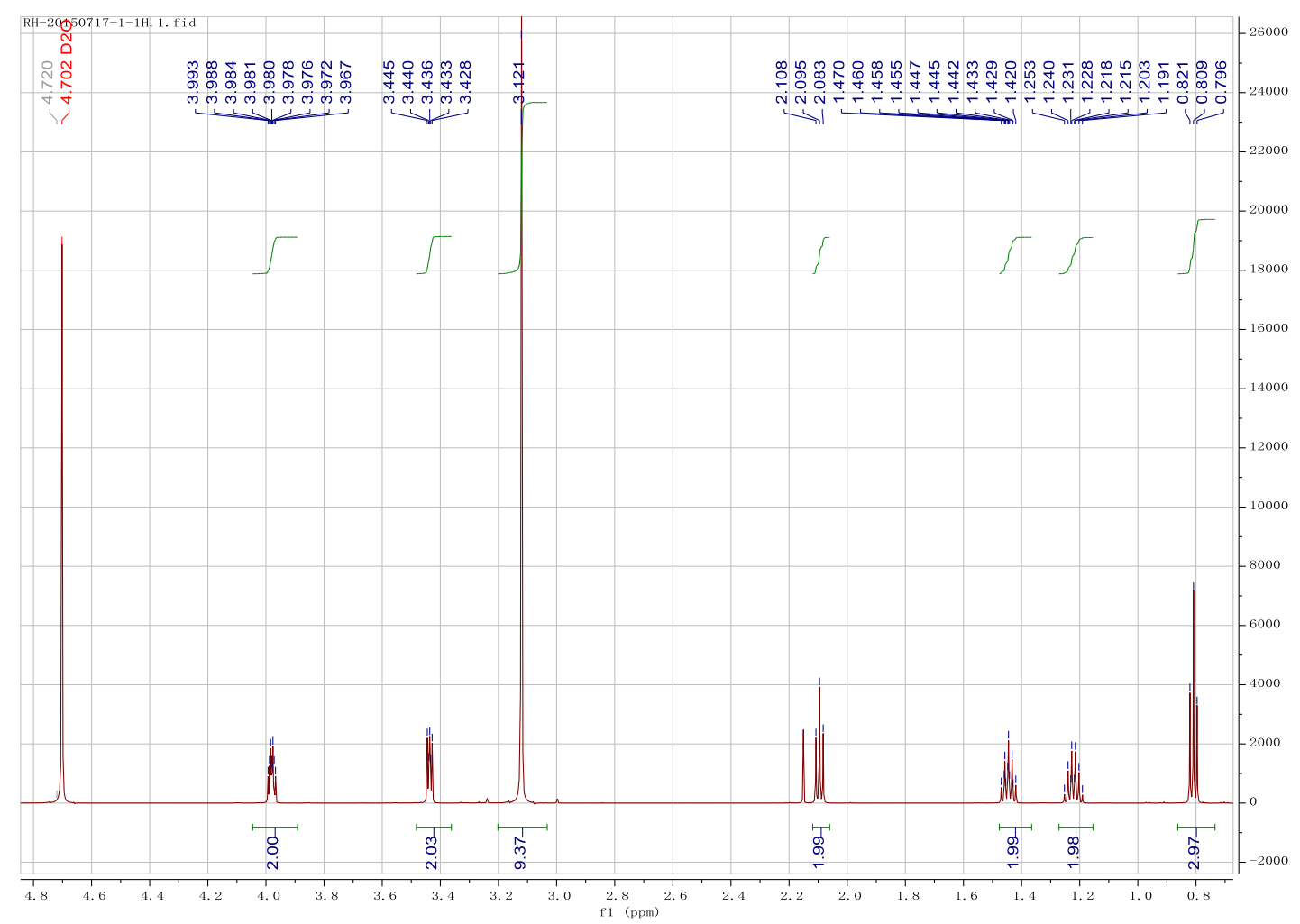

[Ch][Pen]. ${ }^{1} \mathrm{H}$ NMR $\left(600 \mathrm{MHz}, \mathrm{D}_{2} \mathrm{O}\right): 0.81\left(\mathrm{t}, J=6.0 \mathrm{~Hz}, 3 \mathrm{H}, \mathrm{CH}_{3}\right), 1.19-1.25\left(\mathrm{~m}, 2 \mathrm{H}, \mathrm{CH}_{2}\right), 1.42-$ $1.47\left(\mathrm{~m}, 2 \mathrm{H}, \mathrm{CH}_{2}\right), 2.10$ (t, J=7.8 Hz, 2H, CH$), 3.12\left(\mathrm{~s}, 9 \mathrm{H}, \mathrm{CH}_{3}, \mathrm{CH}_{3}, \mathrm{CH}_{3}\right), 3.43-3.44(\mathrm{~m}, 2 \mathrm{H}$, $\left.\mathrm{CH}_{2}\right), 3.97-3.99\left(\mathrm{~m}, 2 \mathrm{H}, \mathrm{CH}_{2}\right)$

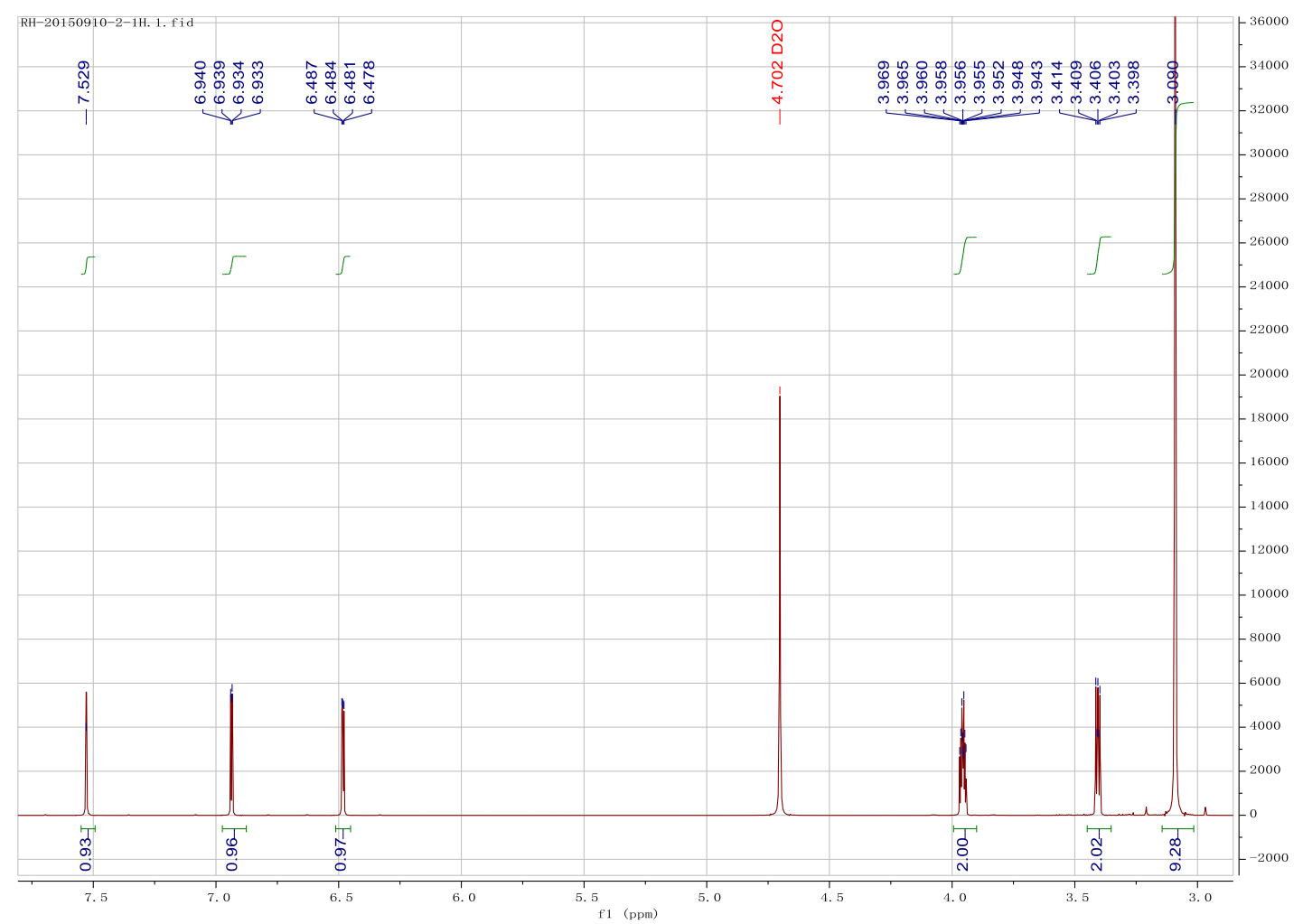

[Ch][Fur]. ${ }^{1} \mathrm{H}$ NMR (600 MHz, $\mathrm{D}_{2} \mathrm{O}$ ): 3.09 (s, 9H, $\left.\mathrm{CH}_{3}, \mathrm{CH}_{3}, \mathrm{CH}_{3}\right), 3.40-3.41$ (m, 2H, $\left.\mathrm{CH}_{2}\right), 3.94$ $-3.97\left(\mathrm{~m}, 2 \mathrm{H}, \mathrm{CH}_{2}\right), 6.48(\mathrm{dd}, J=1.8,3.6 \mathrm{~Hz}, 1 \mathrm{H}, \mathrm{CH}), 6.94(\mathrm{dd}, J=1.8,3.6 \mathrm{~Hz}, 1 \mathrm{H}, \mathrm{CH}), 7.53(\mathrm{~s}$, $1 \mathrm{H}, \mathrm{CH})$ 